\title{
An Efficient Method to Reduce an MAI's Effects in Spectral Amplitude Coding OCDMA Network
}

\author{
Kamal E. Amaseb, Hassan Y. Ahmed, and Medien Zeghid
}

\begin{abstract}
Multiple Access Interference (MAI) in SpectralAmplitude Coding Optical Code Division Multiple Access (SAC-OCDMA) scheme hardly limits the system performance. This problem increases proportionally with the amount of concurrent users. In addition, phase induce intensity noise (PIIN) coming from spontaneous emission process of light source is extra impairment leads to system drop needs to be tackled too. Towards conquer the specified problems, Vectors Combinatorial (VC) codes which based on grouping of certain vectors is proposed. Any positive integer number can be used in both weighs and user's parameters in code building procedure, these technique nominees our code to be a potential reliable applicant for future optical communication schemes. Such flexibility is an exceptional property for $\mathrm{VC}$ compared to SAC-OCDMA counterparts' codes. Compared with the systems employing Hadamard, Modified Frequency Hopping (MFH), Modified Quadratic-Congruence (MQC), and Modified Double Weight (MDW), numerical results show that, VC is effective to reduce the power of MAI and PIIN. It has been exposed that, performance can be superior significantly when VC is used
\end{abstract}

Index Terms - Vectors Combinatorial (VC); Multiple Access Interference (MAI); Spectral-Amplitude Coding Optical Code Division Multiple Access (SAC-OCDMA); Bit Error Rate (BER); PIIN; SNR.

\section{INTRODUCTION}

Multiple access modes are a prerequisite for optical systems and wireless network, which permit several of users to access the network together by sharing a transmission medium and using bandwidth aggressively. CDMA is used widely in wireless networks [1]. The excellent done of CDMA in wireless networks, motivate the telecommunication community to examine the conceivable of CDMA implementation in the optical field. Optical CDMA inherited the CDMA benefits, (which include security and spectral efficiency) and Provide asynchronous data transfer for simple network monitoring and management. OCDMA designed for the future optical fibre networks and permits managers to the data routing without concerning complicated multiplexers and de-multiplexers [1]. OCDMA has attracted considerable research attention for the future access network because of its protocol sensitiveness, high network scalability, no packet crash [2]. (SAC-OCDMA) is easy, asynchronous, economically, powerfully and flexible encoding method that can quell

Published on June 1, 2017.

K. E. A., H. Y. A., and M. Z. are with Faculty of Electrical Engineering Wadi Addwasir Prince Sattam Bin Abdalaziz University, KSA. (e-mail: amaseb9731@hotmail.com, medienzeghid@gmail.com) multiple access interference (MAI) that can be completely removed by spectral coding existent in traditional systems [1].(MAI) is considered as a dominating system-degradation factor for the OCDMA networks. In (SAC-OCDMA) scheme, the impact of MAI can be diminutive by utilizing codes through fixed in-phase cross-correlations [3]. Let us define $\lambda=\sum_{\mathrm{i}-1}^{\mathrm{L}} \mathrm{x}, \mathrm{y}$. As the in-phase cross correlation of two diverse code sequence $\mathrm{X}=(\mathrm{x} 1, \mathrm{x} 2, ., \mathrm{xL})$ and $\mathrm{Y}=(\mathrm{y} 1, \mathrm{y} 2$, ..., yL). However, codes with perfect cross correlation have been considered extensively in [6] -[11], conversely, several of these codes have extremely poor cross correlation (e.g. Hadamard code), or limited obtainable codes number (e.g. Prime number, prime power, even and odd natural number for( MQC, MFH, MDW, EDW, EMD and DDW codes respectively). Furthermore, the potential noise created by multi users aimed to share the medium concurrently will be limited' the OCDMA performance, stated of the MAI. VC code is one of the famous methods that are used to prevent the MAI. Recount studied showed that, the cross correlation properties are not the main issues in building codes, the code length very important for addressing well. When the Long of code length is a disadvantage the code requisite either very wide or narrow filters of bandwidths. When the code is short, it has a limited range of choice. Thus, equalization between the number of code words and code lengths should be completed. VC code exists for applying code length that is neither very long code nor very short.

\section{LAYOUT}

The new submitted code in this paper is a family of $[0,1]$ which is characterized by $(\mathrm{L}, \mathrm{N}, \mathrm{W}, \lambda \mathrm{c})$ when $\mathrm{L}$ is the length, $\mathrm{N}$ is the number of users, $\mathrm{W}$ is the weight (number of ones) and $\lambda c$ is the cross correlation. There many benefits of the proposed VC code family, we can show as follows:

1) Can be used any positive integer number of weights.

2) More flexible for number of users.

3) Simple code construction.

The rest of the paper is organized as follows. In Section III described the family of newly built codes. In Section IV, display the analytical results of system performance. The code properties debated from view of contrast in section $\mathrm{V}$. We have the conclusion in Section VI.

\section{VC CODE DEVELOPMENT}

\section{A. Definition}

Euclidean vector is a geometric object that has both a magnitude (and length) and direction; the size of the vector is the distance between the two points where an initial point $A$ connecting with a terminal point $B$. Each vector has only two points $(A$ and $B)$ where two " $1 \mathrm{~s}$ " will be placed in $A$ and 
$B$ positions respectively. In Math's, normal basis of the wdimensional Euclidean space $\mathrm{R}^{\mathrm{W}}$ is the basis acquired by taking the $W$ basis vectors: $\{V \mathrm{i}: 1 \leq \mathrm{i} \leq W\}[13]$

Where vector $V_{i}$ with is equal 1 in the $\mathrm{i}^{\text {th }}$ form and 0 In another place. E.g., the standard basis for $\mathrm{R}^{3}$ (i.e., $W=3$ ) is given by the vectors as [13]:

$$
\begin{aligned}
\overrightarrow{V_{1}} & =\left[\begin{array}{l}
1 \\
\mathbf{0} \\
\mathbf{0}
\end{array}\right] \\
\overrightarrow{V_{2}} & =\left[\begin{array}{l}
0 \\
1 \\
\mathbf{0}
\end{array}\right] \\
& \vec{V}
\end{aligned}
$$

Fig. 1. A general matrix of $\mathrm{VC}$ when $N=W+1$

The new (VC) code can be designed by isolating the code classification into three stages which are stage one builds vector, stage tow put the vector in the group and the last one is mapping technique [14].

$$
\overline{V_{(i, i+1)}}=\left[\begin{array}{l}
1 \\
0 \\
\cdot \\
\cdot \\
1
\end{array}\right]
$$

\section{B. Stage 1: Building the Vector}

In this Stage, first we built a pillar vector which has two " $1 \mathrm{~s}$ " when the cross correlation equivalent 1 . Second, we let pillar vector $V_{(\mathrm{i}, \mathrm{i}+1)}$ whose $\mathrm{i}^{\text {th }}$ element is "1" and in the last one we put others zero and length equals $\mathrm{N}$ [14].

\section{Stage 2: Putting the Vector in Group}

When the weight $(W)$ value in the row (which is called user) arranged make the in-phase cross correlation (CC) precisely equals 1 for every column, all vector in Fig. 1 is guided as $V_{(i, i+1)}$ for $i$ fixed to user arrangement and $i+1$ shifts to the down by one up to $N$ to make the CC with $N-1$ is accurately equals to 1 (i.e., for $N=4, i=1 ; V_{(\mathrm{i}, \mathrm{i}+1)}=V_{12}$, $V_{13}, V_{14}$; for $N=4, i=2, V_{(i, i+1)}=V_{23}, V_{34}$; for $N=4, i=3, V_{(\mathrm{i}}$, $\mathrm{i}+1)=V_{34}$ ) which means $i$ denotes number of row (user). After combining all column vectors in Fig. 1 , we have to create $(0$, 1) sequence. To be more perfect we can perceive that each column vector holds two " $1 \mathrm{~s}$ "; the number of " $1 \mathrm{~s}$ " per row is represented by $W$; in case the number of users is represented by $\mathrm{N}$, the sequence $\left(\mathrm{V}_{12} \mathrm{~V}_{13} \quad \ldots \mathrm{V}_{1 \mathrm{~N}}\right)\left(\mathrm{V}_{23} \mathrm{~V}_{24}\right.$ $\left.\ldots \mathrm{V}_{2 \mathrm{~N}}\right) \ldots\left(\mathrm{V}_{(\mathrm{N}-1) \mathrm{N}}\right)$ gives a code perfect in-phase CC $(\lambda=1)$ called Ideal Case (IC).

\section{Stage 2: Mapping technique}

when $N-1=W$ in this case it content limited users, the Ideal Case (IC) can be built simply using a column vector. To overcome this problem, we applied a mapping technique (see Fig. 2). The mapping technique mechanism used in order to add more number of users behind the basic number of users given by the basic matrix for an exact weight [7]. Subsequently, $N$ can be written.

$$
N=P(W+1)+R
$$

Clarify number of $(W+1)$ recurrent in diagonal method where $P, R$ is positive integer numbers, and the outstanding users after unit separation for $N$ correspondingly, and $R$ can be expressed as: [13]

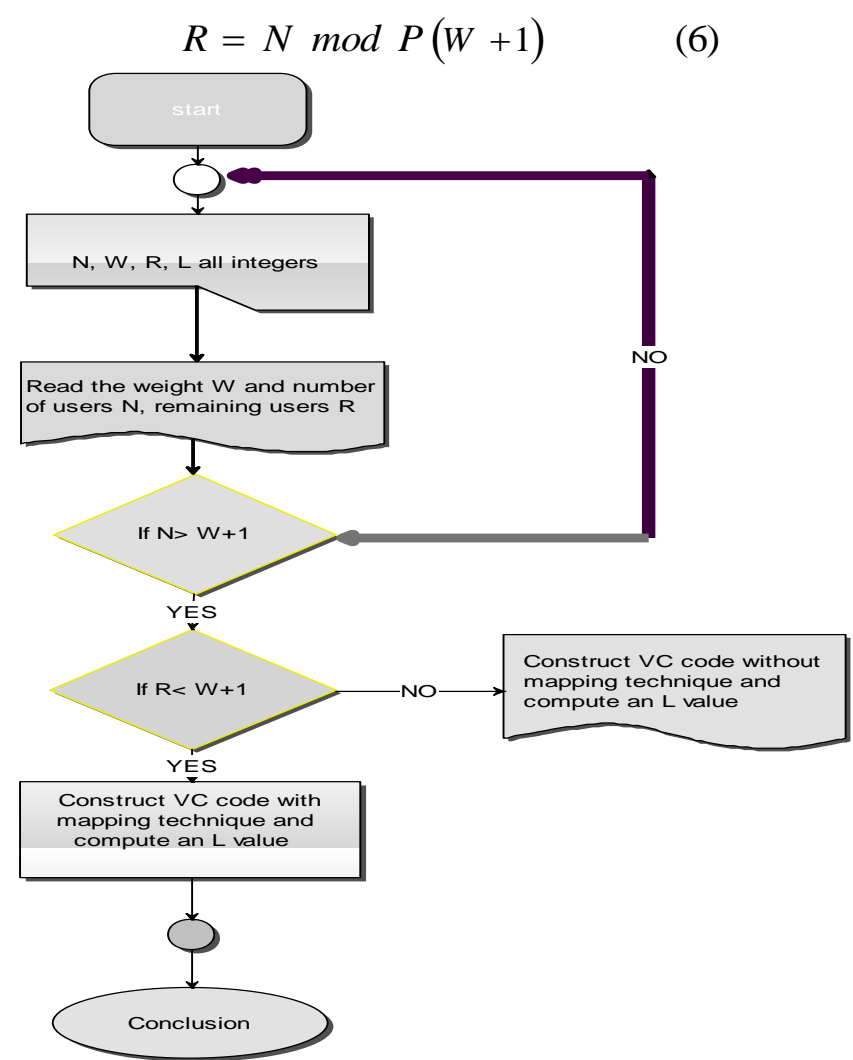

Fig. 2. The major steps and points for VC code family construction

1. Start.

2. Declare variables.

3. Initialize total value $=0$.

4. Get a number from the user.

5. If the number is not equal to -1 , go to step 6 , otherwise go to step 7.

6. Calculate sum.

7. Display sum of numbers.

8. End.

First, go over weight $\mathrm{W}$, number of users $\mathrm{N}$, and remaining users $\mathrm{R}$. If $\mathrm{N}>\mathrm{W}+1$ satisfied, $\mathrm{VC}$ code without mapping technique will be constructed. The parameters $(\mathrm{W}$, R) have to be added if the state $\mathrm{N}>\mathrm{W}+1 \& \& \mathrm{R}<\mathrm{W}+1$ is mollified (see Fig. 3). To clarify (5) and (6) where mod appears modulo division, let us think through the example $\mathrm{N}=6, \mathrm{~W}=2$, substitute the values in (5), (6), gives $6=2 \times$ $(2+1)+0$, which means $\mathrm{P}=2$ and $\mathrm{R}=0$. For $\mathrm{N}=6, \mathrm{~W}=3$, gives 
$6=1 \times(3+1)+2$ which means $\mathrm{P}=1$ and $\mathrm{R}=2$.

A mapping technique have apply to added more number of users in VC code family, (see Fig. 3) Which are works by diagonally repeating the IC for the $(W+1)$ users $P$-times and filling the void with zeros. The length of that section is P. $\frac{\mathrm{W}(\mathrm{W}+1)}{2}$. Consequently, an IC with the parameters $(W$, $R$ ) have to add if $R<W+1$ is satisfied (i.e., $R=N \bmod P(W+1)$ $\neq 0$ ) (see Fig. 3). The length of that second section of the code is $\underline{\mathrm{R} \times(2 \mathrm{~W}-\mathrm{R}+1)}$. Finally, the whole length $L$ is given by:

$$
\mathbf{L}=\frac{W N+R(w+1-R)}{2}
$$

Fig. 3. Graphic representation of mapping techniques for $N=P(W+1)+R$

In Table I, $N=10 \& W=4$ by using (5), (6) gives $10=2 \times$ $(4+1)+2$, resulting $P=2$ and $R=2$ that means we should repeated (9) tow time.[13]

$(P=2)$ in diagonal fashion, resulting to 8 users and add two other users $(R=1)$ after user\#8 in diagonal fashion also. By using (12) $L=10 \times 4+2(4-1+2) / 2$, the code length will be 17. In Table I, there are three clusters of codes, the first one (first six columns) is the $\mathrm{VC}$ code with the parameter value $(3,4)$ which means $W=4$ and $N=4$ having $C C$ equal to 1 ; the second (from columns 7 to 12) is a duplication from the first one with the parameters $(3,4)$; the third cluster (from columns 13 to 17$)$ is the VC with the parameters $(3,2)$ which means $W=4$ and $N=2($ i.e., $R=N$ ) since the condition $R<W+1$ $(2<4)$ is contented having CC matches to resistor [13].

TABLE I: VC CODE FOR $\mathrm{N}>\mathrm{W}+1 \& \& \mathrm{R}<\mathrm{W}+1$

\begin{tabular}{llllllllllllllllllll}
\hline \hline 1 & 1 & 1 & 1 & 0 & 0 & 0 & 0 & 0 & 0 & 0 & 0 & 0 & 0 & 0 & 0 & 0 & 0 & 0 & 0 \\
\hline 1 & 0 & 0 & 0 & 1 & 1 & 1 & 0 & 0 & 0 & 0 & 0 & 0 & 0 & 0 & 0 & 0 & 0 & 0 & 0 \\
\hline 0 & 1 & 0 & 0 & 1 & 0 & 0 & 1 & 1 & 0 & 0 & 0 & 0 & 0 & 0 & 0 & 0 & 0 & 0 & 0 \\
\hline 0 & 0 & 1 & 0 & 0 & 1 & 0 & 1 & 0 & 1 & 0 & 0 & 0 & 0 & 0 & 0 & 0 & 0 & 0 & 0 \\
\hline 0 & 0 & 0 & 1 & 0 & 0 & 1 & 0 & 1 & 1 & 0 & 0 & 0 & 0 & 0 & 0 & 0 & 0 & 0 & 0 \\
\hline 0 & 0 & 0 & 0 & 0 & 0 & 0 & 0 & 0 & 0 & 1 & 1 & 1 & 1 & 0 & 0 & 0 & 0 & 0 & 0 \\
\hline 0 & 0 & 0 & 0 & 0 & 0 & 0 & 0 & 0 & 0 & 1 & 0 & 0 & 0 & 1 & 1 & 1 & 0 & 0 & 0 \\
\hline 0 & 0 & 0 & 0 & 0 & 0 & 0 & 0 & 0 & 0 & 0 & 1 & 0 & 0 & 1 & 0 & 0 & 1 & 1 & 0 \\
\hline 0 & 0 & 0 & 0 & 0 & 0 & 0 & 0 & 0 & 0 & 0 & 0 & 1 & 0 & 0 & 1 & 0 & 1 & 0 & 1 \\
\hline 0 & 0 & 0 & 0 & 0 & 0 & 0 & 0 & 0 & 0 & 0 & 0 & 0 & 0 & 1 & 0 & 1 & 0 & 1 & 1
\end{tabular}

\section{SySTEM PERFORMANCE}

We can compute the Signal to Noise Ratio (SNR) by using the similar in [10]-[11] when we used the different codes. The phase induced intensity noise, the photodiode shot noise and the thermal noise are taken into account. Signal to noise ratio (SNR) of the VC code family is given by:

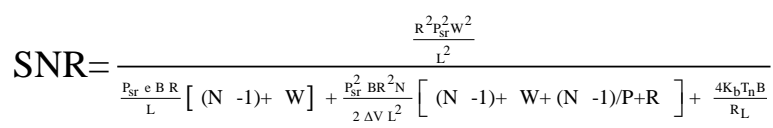

where $\mathfrak{R}$ is the photodiode reacting, $P_{s r}$ is the efficacious power of a broadband source at the receiver, $e$ is the electronic charge, $B$ is an tantamount noise bandwidth of the receiver, $K_{B}$ is the Boltzmann's constant, $T_{n}$ is the Absolute receiver noise temperature, $R_{L}$ is the receiver load resistor, $\Delta V$ is the optical source bandwidth, $W, N, L, P$ and $R$ are the code weight, the number of users, the number of mappings, the code length and the remaining of users after modulo operation respectively as being the parameters of VC itself . The Bit Error Rate (BER) is computed from the SNR using the Gaussian approximation as [11]-[12].

$$
\mathrm{BER}=0.5 \mathrm{erfc} \sqrt{S N R / 8}
$$

TABLE II: THE SUBSEQUENT PARAMETER OF (VC) [7]-[10]

\begin{tabular}{lllllll}
\hline \hline $\begin{array}{l}\text { Subsequent } \\
\text { parameters }\end{array}$ & $\Delta V$ & $B$ & $T n$ & $R_{L}$ & $\eta$ & $\Lambda$ \\
\hline & 3.75 & 1.625 & 300 & 1030 & 0.6 & $1550 \mathrm{~nm}$ \\
& $\mathrm{THz}$ & $\mathrm{GHz}$ & $K$ & $\Omega$ & & \\
\hline \hline
\end{tabular}

\section{COMPARISON AND EVALUATION}

We use the ideal in phase CC to complete a series of code family, for comparison, some properties of the code like for example VC code, MQC, MDW, MFH, EMD, DDW and Hadamard, which are registered in Table III. It is shown that VC codes exist for all even, odd and prime positive integer, while MDW codes are existing for even $n$ weights, Hadamard codes are existing only when the weight is $2 \mathrm{~m}-1$ where $\mathrm{m} \geq 2$, MQC and MFH are existing for a prime number $\mathrm{p}$ and a prime power $\mathrm{q}$ given by $\mathrm{q}=\mathrm{pn}$ where $\mathrm{n}$ is a positive integer correspondingly. The table also displays that, the VC codes have the best cross correlation, while Hadamard codes when the number of users increased have increasing value of cross correlation.

Fig. 4 demonstrations the relative among the number of active users and the SNR for VCC when the parameters used in the VCC codes building are $W=4, \mathrm{P}=2, \mathrm{R}=1$. The SNR for MQC, Hadamard and MFH are also displayed as a reference with the parameters $p=13, q=16$ and code length $\mathrm{N}=256$ for MQC, Hadamard codes and MFH respectively. 
TABLE II: SAC-OCDMA CODES COMPARISONS

\begin{tabular}{|c|c|c|c|c|c|c|}
\hline $\begin{array}{l}\text { Code } \\
\text { family }\end{array}$ & Existence & Weight & Size & $\Lambda$ & Code length & SNR \\
\hline \multirow{2}{*}{ MQC } & \multirow{2}{*}{ Primes $p>2$} & \multirow{2}{*}{$p+1$} & \multirow{2}{*}{$p^{2}$} & \multirow{2}{*}{1} & \multirow{2}{*}{$p^{2}+\mathrm{p}$} & $\Delta v(p+1)$ \\
\hline & & & & & & $B K[(K-1) / p+p+K]$ \\
\hline \multirow{2}{*}{ EMD } & \multirow[b]{2}{*}{ All GF } & & \multirow{2}{*}{ w } & \multirow{2}{*}{1} & \multirow{2}{*}{$K+[K(W-2)+1)$} & {$[1] \quad\left[\mathrm{R} \mathrm{P}_{\mathrm{st}} \mathrm{W} / \mathrm{N}\right] 2$} \\
\hline & & & & & & $\overline{\mathrm{eBRP}_{\mathrm{st}} \mathrm{W} / \mathrm{N}+4 \mathrm{Kk}_{\mathrm{b}} \mathrm{T}_{\mathrm{n}} \mathrm{B} / \mathrm{R}_{\mathrm{l}}}$ \\
\hline \multirow{2}{*}{ MDW } & \multirow{2}{*}{$\begin{array}{c}\text { Even integer } \\
n\end{array}$} & \multirow{2}{*}{$\begin{array}{l}\text { Any number even } \\
\text { greater than } 2\end{array}$} & \multirow{2}{*}{$\mathrm{N}_{\mathrm{B}}$} & \multirow{2}{*}{1} & \multirow{2}{*}{$\mathrm{K}_{\mathrm{B}}$} & {$[4-5] \quad 2\left(\frac{W}{\lambda}-1\right) \Delta v$} \\
\hline & & & & & & $B K\left[\left(\frac{K}{2}+\frac{W}{\lambda}-2\right]\right.$ \\
\hline \multirow{2}{*}{ DDW } & \multirow{2}{*}{ Even integer } & \multirow{2}{*}{2} & \multirow[b]{2}{*}{$\mathrm{w} / 2+1$} & \multirow[b]{2}{*}{1} & \multirow[b]{2}{*}{$\mathrm{K}+1$} & [3] $\frac{\mathrm{R}^{2} \mathrm{P}_{\mathrm{sr}}^{2} \mathrm{~W}^{2}}{\mathrm{~N}^{2}}(\mathrm{~W}-1)^{2}$ \\
\hline & & & & & & $\frac{2 \mathrm{eBRP}_{\mathrm{SR}} \mathrm{W}}{\mathrm{N}}(\mathrm{W}-1)+\frac{\mathrm{R}^{2} \mathrm{BP}_{\mathrm{s}}^{2} \mathrm{KW}^{2}}{2 \mathrm{~N}^{2} \Delta \mathrm{v}}(\mathrm{W}-1)+\frac{4 \mathrm{~K}_{\mathrm{b}} \mathrm{T}_{\mathrm{n}} \mathrm{B}}{\mathrm{R}_{\mathrm{L}}}$ \\
\hline \multirow{2}{*}{$\begin{array}{l}\text { Hadamar } \\
\text { d }\end{array}$} & \multirow{2}{*}{$m \geq 2$} & \multirow{2}{*}{$2^{m-1}$} & \multirow{2}{*}{$2^{m}-1$} & $2^{m-}$ & \multirow{2}{*}{$2^{m}$} & \multirow{2}{*}{ [9] $\frac{\Delta v}{B K(K+1)}$} \\
\hline & & & & 2 & & \\
\hline $\mathrm{VC}$ & $N>W+1$ & Positive integer & $\begin{array}{c}\text { Any } \\
\text { number of } \\
\text { users }\end{array}$ & 1 & $(W N+R(W+1-R)) / 2$ & Refer to Eq (8) \\
\hline
\end{tabular}

In Fig. 4, the power user is $-10 \mathrm{dBm}$ which is concentration taking into account the impacts of power noise, shot noise and thermal noise. It is shown that the VCC code allows SNRs much greater than MFH, Hadamard and MQC, with the great values of $P$ and $R$ higher SNR can be achieved with high number of active users for small weight.

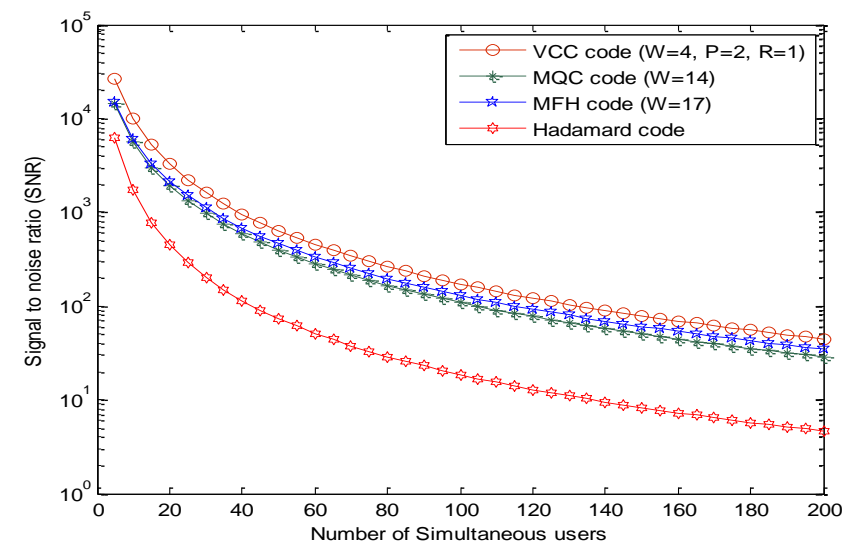

Fig. 4. SNR versus the number of simultaneous users

In Fig. 5, shows the BER is in contrast to the number of working users when Psr $=-10 \mathrm{dBm}$., we can keep an eye the lower BER of VC code than MQC, MFH and Hadamard codes even for lower value of $W=4$ compared to $W=14$, $W=17$. This is due to good arrangement of VC code building using mapping techniques which reduces the MAI and PIIN. It has obviously shown that the system using $\mathrm{VC}$ code is extremely low BER than that using MQC, MFH and Hadamard consequent to perfect properties of $\mathrm{VC}$ code design which decreases the MAI and PIIN.

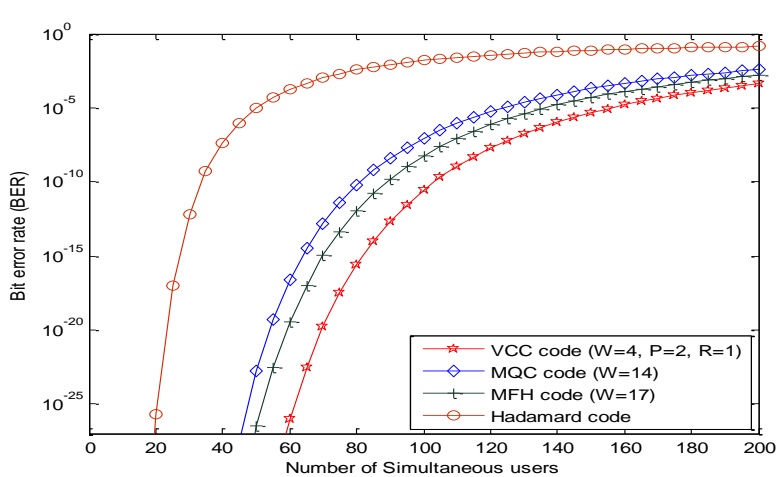

Fig. 5. BER versus the number of active users

Fig. 6 shows the BER variations with effective power Psr when $W=14, \mathrm{P}=2$ and the number of active users is 30 . Also shown the performance of the former system using MQC $(W=14)$ and $\mathrm{MFH}(W=17)$ for comparison. In this figure, we have concentration taking into account the impacts of the intensity noise, thermal noise and shot noise at $2.5 \mathrm{~Gb} / \mathrm{s}$. It has shown that, the VC code gives a much better performance when the effective power Psr is large (when Psr $>-18 \mathrm{dBm}$ ). This due to elimination of the MAI effects through cross correlation controls by using mapping techniques [6]. At the lower values of Psr (when Psr < $18 \mathrm{dBm})$, the performance of the system for all codes is nearly the same. 


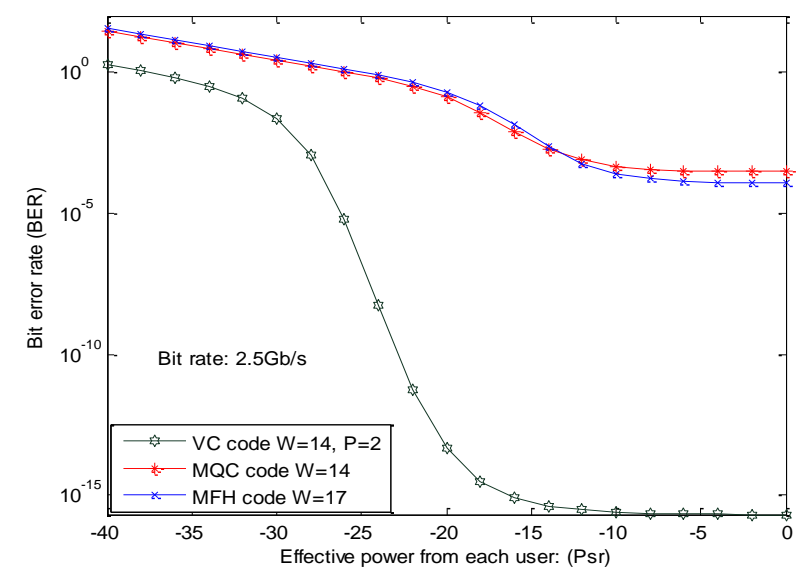

Fig. 6. BER versus effective source power Psr with 30 active users at 2.5 $\mathrm{GB} / \mathrm{s}$

Fig. 7 displays the PIIN plotted against the received power for the VC, MQC and MFH uses the parameters: $\mathrm{W}=4,12$ and 10 for $\mathrm{VC}, \mathrm{MQC}$ and $\mathrm{MFH}$ respectively at data rate $2.5 \mathrm{~GB} / \mathrm{s}$. We can see that when the received power increases from the figure, the PIIN noise for all the codes increases linearly. The PIIN noise of VC code family is less compared to that of MQC and MFH codes. Also the PIIN noise can be successfully suppressed by using VC code family. This is because by using mapping techniques, the interference power from other users is reduced by the increase of the code length, and finally eliminates the MAI effects.

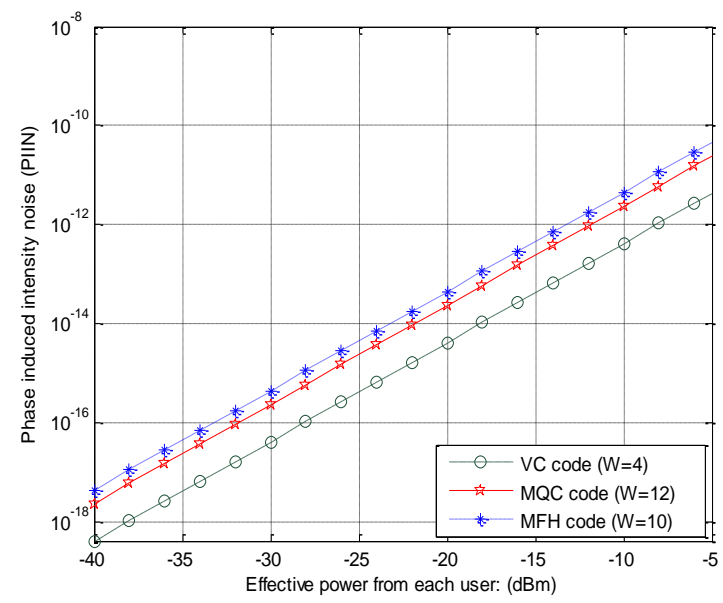

Fig. 7. PIIN Noise versus Number of Active Users for VC, MQC and MFH codes at data rate $2.5 \mathrm{~Gb} / \mathrm{s}$ for different values of $\mathrm{W}$.

The received power and corresponding eye pattern diagram for VC codes are shown in Fig. 8. The eye graph illustrated in Fig. 8 (b) clearly explains that the VC code gives enhanced performance, having a bigger eye opening. The vertical space between the highest of the eye opening and maximum signal level gives the degree of disfigurement. The more the eye closes the tougher it is to discriminate among $1 \mathrm{~s}$ and $0 \mathrm{~s}$ in the signal. The height of the eye opening at the specified sampling time shows the noise margin or immunity to noise.

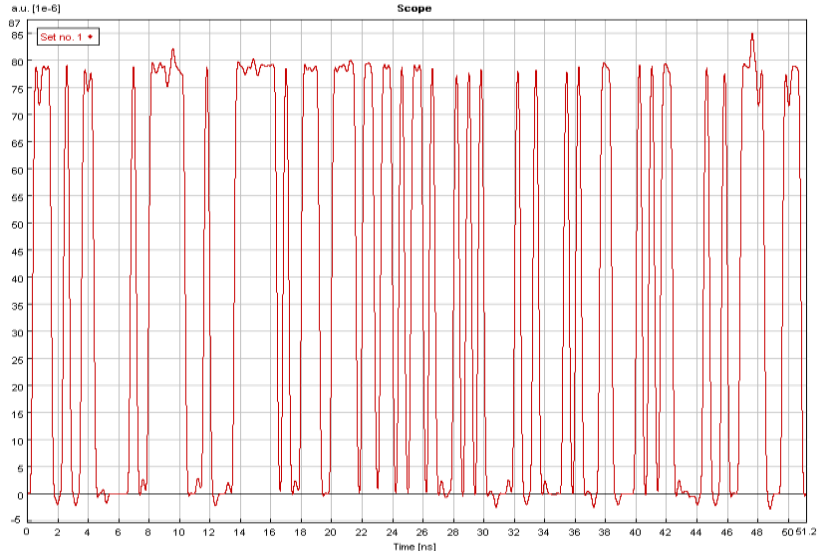

(a)

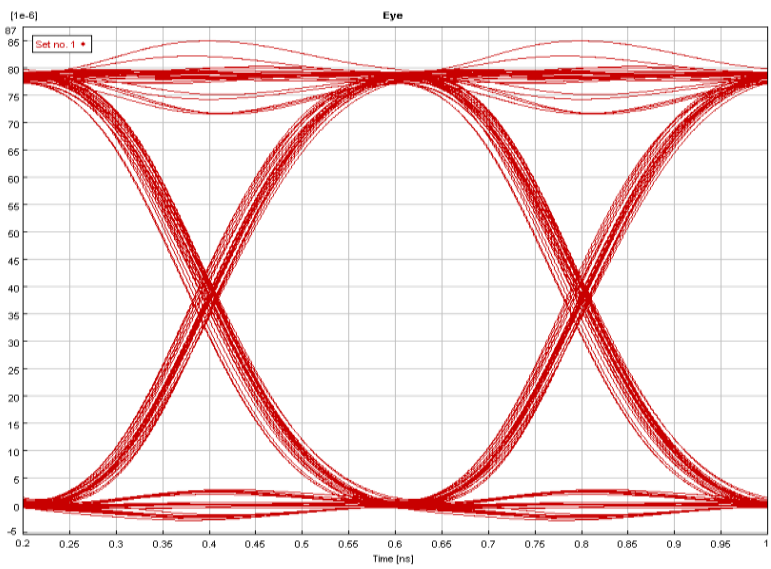

(b)

Fig. 8. Eye diagram for VC code at $10 \mathrm{Gbit} / \mathrm{s}$ for $50 \mathrm{~km}$ (a) NRZ format (b) RZ format

\section{CONCLUSION}

This paper, a presents a new code using ideal in-phase cross correlation (CC) is done. In the vector combination (VC) codes which based on grouping of certain vectors any positive integer number can be used in both weights and user parameters in code building procedure. This code is discussed and described with related equations. The outcome of system operation compared with reported codes. The benefit of the VC code can shortly as follows:

1) Large tractability in choosing the number of users than MQC, MFH, MDW, DDW, EMD and Hadamard codes (free cardinality);

2) Good property in cross-correlation control;

3) Short length;

4) It has shown that performance can be improved significantly when VC code used instead of MQC, MFH, MDW, DDW, EMD and Hadamard codes.

\section{REFERENCES}

[1] M. Moghaddasin, G.Mamdoohi, Ahmad Shukr iMuhammad Noor, MohdAdzirMahdi, SitiBarirah AhmadAnas" (Development of SACOCDMA in FSO with multi-wavelength laser source)" Article history: Received8April2015 Received in revised form 9 July2015 Accepted28July2015.

[2] M.Z. Norazimah, Hamza M. R. Al-Khafaji, S. A. Aljunid, and Hilal A. Fadhil School of Computer and Communication Engineering, University Malaysia Perlis (UniMAP), Perlis, Malaysia Corresponding author. (Hamza M. R. Al-Khafaji) (Performance Comparison of Different Detection Techniques in Long-Haul Fiber SAC-OCDMA Systems) 3rd International Conference on Photonics 2012, Penang, 1-3 October 2012.

[3] Simranjit Singh a, , Ramandeep Kaur a, Amanvir Singh a, R.S. Kaler " Novel security enhancement technique against eavesdropper for 
OCDMA system using 2-D modulation format with code switching scheme" Received 8 September 2014 Revised 23 January 2015 Available online 14 February 2015.

[4] K.S. Nisar Numerical construction of generalized matrix partitioning code for spectral amplitude coding optical CDMA systems" Volume 130, February 2017, Pages 619-632

[5] J. A. Salehi, "Code division multiple access techniques in optical fiber network-Par I: Fundamental principles," IEEE Trans. Commun., vol.37, pp. 824-833, 1989 .

[6] J. A. Salehi and C. A. Brackett, "Code division multiple access techniques in optical fibre network-Part II: System performance analysis," IEEE Trans. Commune., vol. 37, pp. 834-842, 1989.

[7] S.A.Aljunid, ,M.Ismail, A.R.Ramli, Borhanuddin M. Ali, and Mohamad Khazani Abdullah, "A New Family of Optical Code Sequences for Spectral-Amplitude-Coding, Optical CDMA Systems" IEEE Photonics Technology Letters, Vol. 16, No. 10, October 2004.

[8] Syed Alwee Aljunid, Zuraidah Zan, Siti Barirah Ahmad Anas and Mohd. Khazani Abdullah, A New Code for Optical Code Division Multiple Access Systems, Malaysian Journal of Computer Science. 17 (2004) 30-39.

[9] Ivan B. Djordjevic and Bane Vasic, "Combinatorial Constructions of Optical Orthogonal Codes for OCDMA Systems". Journal of Lightwave Technology, Vol. 21, September 2003

[10] Zou Wei, H. M. H. Shalaby, H. Ghafouri-Shiraz, "Modified Quadratic Congruence codes for Fiber Bragg-Grating-Based SAC-OCDMA," Journal of Lightwave Technology, Vol. 19, no. 9, pp. 1274-1281, Semptember. 2001

[11] Zou Wei, H. M. H. Shalaby, H. Ghafouri-Shiraz, "Modified Quadratic Congruence codes for Fiber Bragg-Grating-Based SAC-OCDMA," Journal of Lightwave Technology, Vol. 50, August 2002, pp. 12091212.

[12] M. Kavehrad, and D. Zaccarh, "Optical Code-Division-Multiplexed Systems Based on Spectral Encoding of Noncoherent Sources," Journal ofLightwave Technology, Vol. 13, No. 3, March 1995

[13] H. Y. Ahmed, M. Elmaleeh , H. Adnan , and S. Aljunid Department of Electrical Engineering, Salam Bin Abdulaziz University, Kingdom of Saudi Arabia Faculty of Engineering, University of Gezira, Sudan School of Computer and Communication Engineering, Universiti Malaysia Perlies, Malaysia" Optical CDMA: Performance of Spectral-Amplitude Coding with New Direct Recovery Scheme using
Vector Combinatorial (VC) Code" The International Arab Journal of Information Technology, Vol. 10, No. 5, September 2013

[14] H. Y. Ahmed, M. AlaleehL, Hilal Fadhil, S. A. AljunidLJ , Ammar E. Babiker, N.M. Saad "Performance Analysis of Optical Cdma System Using Vc Code Family Under Various Optical Parameters" Journal of Engineering Science and Technology Vol. 7, No. 3 (2012)

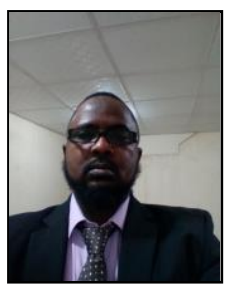

Kamal Elsiddig Amaseb received his B.Eng. In computer engineering and $\mathrm{MSc}$ in computer engineering \& network from Gezira University, Sudan in 2000 and 2008 respectively. Currently, he is a lecturer in the Electrical Engineering Department, College of Engineering, Sattam Bin Abdulaziz University, KSA. His research interests include computer network, wireless communications networks, Optical communications.

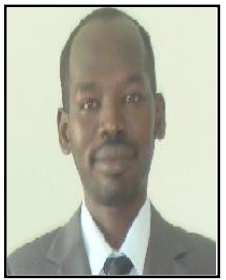

Hassan Yousif Ahmed received his B.Eng. In computer engineering and MSc in computer science and information from Gezira University, Sudan in 2002 and 2007 respectively. In 2010 he received his $\mathrm{PhD}$ degree from the University Tecknolg PETRONAS Department of Electrical and Electronic Malaysia. Currently, he is an associate professor in the Electrical Engineering Department, College of Engineering, Sattam Bin Abdulaziz University, KSA His research interests include computer network, wireless communications networks, optical communications.

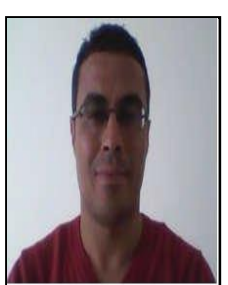

Medien Zeghid received his B.Sc., Microelectronic: Faculty of Sciences of Monastir, Tunisia, June 2003 Grade (Good).M.Sc Faculty of Sciences of Monastir, Tunisia, July 2005. Grade (Excellent) Ph.D., Sciences and Technologies of Information and Communication, Laboratory of Information Science and Technology, Communication and Knowledge, University of Bretagne-Sud in Lorient (France) University of South Brittany (Lorient-France) October 2011 ssistant professor, Prince Sattam Bin Abdulaziz University, College of Engineering, Department of Electrical Engineering, Wadi Addwasir- Kingdom of Saudi Arabi 